\title{
Finding the Sum of Two Numbers from Their Difference
}

\author{
Pirooz Mohazzabi*, Thomas A. Fournelle \\ Department of Mathematics and Physics, University of Wisconsin-Parkside, Kenosha, WI 53141, USA. \\ * Corresponding author. Tel.: (262) 595-2529; email: mohazzab@uwp.edu \\ Manuscript submitted December 12, 2016; accepted February 13, 2017. \\ doi: 10.17706/ijapm.2017.7.3.
}

\begin{abstract}
In a one dimensional random walk of $N$ steps, with a probability $p$ of taking a step to the right, and a probability 1- $p$ of taking a step to the left, it is not possible to determine from the ending position of the walk what $N$ and $p$ are. However, if the $N$-step random walk is repeated many times then a statistical analysis of the ending positions can be used to estimate both $N$ and $p$. In this article we present the details of this analysis and test the results by Monte Carlo simulations. These simulations show that very accurate estimates of $N$ and $p$ are obtained even when the number of experimental trials is relatively small.
\end{abstract}

Key words: Sum from difference, two numbers, probability, random walk, simulation.

\section{Introduction}

The general question that we will try to answer in this article is the following: Consider an experiment in which an unknown number $(N)$ of objects is randomly divided into two parts with probabilities $p$ and 1-p, resulting in $N_{1}$ and $N_{2}$. This can be, for example, a random walk in one dimension, flipping a given number of coins, or randomly throwing a number of balls into two bins. At the end of the experiment, only the difference of the two numbers $N_{1}-N_{2}$ is given to us and nothing else. The question is, can we calculate the sum of the two numbers $N_{1}+N_{2}=N$ from this information? Of course the answer is no, because there are infinitely many sums $N_{1}+N_{2}$ with the same difference $N_{1}-N_{2}$. In other words, there is seemingly no information in $N_{1}-N_{2}$ about $N_{1}+N_{2}$. But what if the experiment is repeated with the same unknown total number of objects? In other words, suppose we ask a question and receive absolutely no information about the answer. Does it make sense to keep asking the very same question again and again? Although it seems counter-intuitive, the answer is sometimes yes. And, as we shall see in what follows, we will be able to find not only the sum of the two numbers but also the probabilities of their occurrences very accurately from their difference when the experiment is repeated many times.

As a specific example, consider two bins and suppose someone randomly throws a number of balls into them. At the end of the process, we are given the difference of the number of balls in the bins, $N_{1}-N_{2}$. The question is, from this information can we determine the total number of the balls $N$ thrown into the bins and the probability with which they were thrown into each bin? The first part of the question is asking for the sum $N_{1}+N_{2}$ when we know only the difference $N_{1}-N_{2}$, which is, of course, impossible to answer. And, finding the probabilities with which the bins received the balls is equally impossible. However, it turns out that if this same process is repeated a number of times, eventually we will find the answer. This is very interesting and yet counter-intuitive because although the result of each trial seemingly contains no information about what we are looking for, when repeated enough times the trials yield complete information. 
A number of mathematicians and statisticians with whom these results were shared were extremely doubtful that any of this could be true until they saw the mathematical proof and the Monte Carlo simulations. The reason for such skepticism, of course, is that there are infinitely many sums $N_{1}+N_{2}$ for which the difference $N_{1}-N_{2}$ is the same. This, however, misses the point. Although the difference of two numbers virtually provides no information about their sum, for non-negative numbers $N_{1}$ and $N_{2}$ we have $\left|N_{1}-N_{2}\right| \leq N_{1}+N_{2}$. Therefore, although not so obvious, there is a very small amount of latent information buried within the difference of the two numbers about their sum. This information accumulates when the process is repeated and eventually the full picture emerges.

Based on the above observations, in principle, one may compute many differences and take the largest in absolute value as an estimate of $N_{1}+N_{2}$. This, however, is very inefficient because to obtain the actual sum in this process, at least in one of the trials all the balls should go into one bin. However, the probability of such an event is extremely small if the number of trials is fairly large. For example if 20 balls are randomly distributed between two bins, the chance of all the balls going into one bin (assuming a probability of 0.5 for each bin) is

$$
p=\frac{2}{2^{20}}<2 \times 10^{-6}
$$

Therefore, it is highly unlikely for the event to happen.

On the other hand, as we show in the following statistical analysis, it turns out that when the mean value of the difference of the two numbers is combined with its variance, it provides an extremely efficient algorithm for estimating not only $N_{1}+N_{2}$ but also the probability with which the balls are thrown into the bins. Thus, the seeming paradox of solving a problem with no useful information is not really a true paradox at all, but only an underestimation of the amount of information we are really being given in each trial.

\section{Theory}

Random walk is a stochastic process that is found in many areas, ranging from science to finance [1], [2] Consider a one-dimensional random walk along the $x$ axis, starting at the origin [3]. All steps are of the same length, which is taken to be the unit of length. The position of the walker after $N$ steps is

$$
x=\sum_{i=1}^{N} s_{i}
$$

where $s_{i}= \pm 1$. The position of the walker can also be written as

$$
x=N_{1}-N_{2}
$$

where $N_{1}$ and $N_{2}$ are the total steps in the positive and negative directions, respectively. Let the probability of a step in the positive direction be $p$ and that in the negative direction be 1- $p$. Taking the average of both sides of equation (3), we have

$$
\langle x\rangle=\left\langle N_{1}-N_{2}\right\rangle=\left\langle N_{1}>-\left\langle N_{2}\right\rangle=p N-(1-p) N\right.
$$

or

$$
\langle x\rangle=\left\langle N_{1}-N_{2}\right\rangle=(2 p-1) N
$$

where $\langle\cdots>$ indicates ensemble average.

Squaring both sides of equation (2), we get

$$
x^{2}=\left(\sum_{i=1}^{N} s_{i}\right)^{2}=\sum_{i=1}^{N} s_{i}^{2}+\sum_{i \neq j=1}^{N} s_{i} s_{j}
$$


Each term in the first sum of the right hand side is just 1 . Therefore, the first sum is $N$. The second sum written in expanded form is

$$
\left.\sum_{i \neq j=1}^{N} s_{i} s_{j}=\left(s_{1}+s_{2}+s_{3}+\cdots+s_{N}\right)\left(s_{1}+s_{2}+s_{3}+\cdots+s_{N}\right) \quad \text { (no } s_{i}^{2} \text { terms }\right)
$$

On the average there are $p N$ positive and (1- $p) N$ negative terms in each of the sums in this equation. Each term in the left sum, however, multiplies only $N-1$ term of the second sum. Therefore, the total number of terms of the forms $(+1)(+1),(-1)(-1)$, and $(+1)(-1)$ or $(-1)(+1)$ are

$$
\begin{aligned}
(+1)(+1): & (p N)[p(N-1)] \\
(-1)(-1): & {[(1-p) N][(1-p)(N-1)] } \\
(+1)(-1) \text { or }(-1)(+1): & 2(p N)[(1-p)(N-1)]
\end{aligned}
$$

Therefore, the mean value of equation (6) is

$$
\begin{aligned}
\left\langle\sum_{i \neq j=1}^{N} s_{i} s_{j}\right\rangle=(p N)[p(N-1)]+[(1-p) N][(1-p)(N-1)]-2(p N)[(1-p)(N-1)] \\
=(2 p-1)^{2}(N-1) N
\end{aligned}
$$

Then taking the average of both sides of equation (5) gives

$$
\left\langle x^{2}>=<\left(N_{1}-N_{2}\right)^{2}>=N\left[1+(2 p-1)^{2}(N-1)\right]\right.
$$

Equations (4) and (7) are well known and can be found in essentially any discussion of random walks. For the special case $p=0.5$, these equations reduce to much simpler forms, namely $\langle x\rangle=0$ and $\left\langle x^{2}\right\rangle=\mathrm{N}$, respectively.

Let us now solve equations (4) and (7) simultaneously for $N$ and $p$. The result is

$$
N=\frac{\sigma^{2}}{2}\left(1+\sqrt{1+\frac{4<x\rangle^{2}}{\sigma^{4}}}\right)
$$

and

$$
p=\frac{\langle x\rangle}{\sigma^{2}\left(1+\sqrt{1+\frac{4<x>^{2}}{\sigma^{4}}}\right)}+\frac{1}{2}
$$

where $\sigma^{2}$ is the variance of the trials, given by

$$
\sigma^{2}=\left\langle x^{2}>-\langle x\rangle^{2}\right.
$$

According to equations (8) and (9), therefore, the total number of steps $N$ and the probability of the walk $p$ can be determined from the mean and the variance of $N_{1}-N_{2}$.

Now consider an experiment in which a total number of balls $N$ are randomly thrown into two bins. There is a one-to-one correspondence between this problem and the problem of random walk described above. Consequently, the results obtained for random walk can be mapped to randomly throwing balls into two bins. Thus, a step in the positive direction in the random walk corresponds to throwing a ball in bin number 1 and a step in the negative direction corresponds to throwing a ball in bin number 2 . After each trial the difference of the number of balls in the bins, $N_{1}-N_{2}$, is given to us. The trial is repeated a large number of times, $n$, each time throwing the same total number of balls $N$ and with the same probabilities $p$ and 1- $p$, which we don't know yet. At the end of the experiment, we can calculate the average values of $N_{1}-N_{2}$ and $\left(N_{1}-N_{2}\right)^{2}$ from 


$$
\left\langle x>=<N_{1}-N_{2}>=\frac{1}{n} \sum_{i=1}^{n}\left(N_{1}-N_{2}\right)_{i}\right.
$$

and

$$
<x^{2}>=<\left(N_{1}-N_{2}\right)^{2}>=\frac{1}{n} \sum_{i=1}^{n}\left(N_{1}-N_{2}\right)_{i}^{2}
$$

and the variance $\sigma^{2}$ from equation (10). Then from equations (8) and (9), $N$ and $p$ can be determined. In other words, knowing $N_{1}-N_{2}$ for the individual trials enables us to find not only $N_{1}+N_{2}$, but also the probabilities with which the balls were thrown into the bins, a simple yet rather counter-intuitive result.

Another interesting aspect of these results is the precision with which $N$ and $p$ can be estimated with even a fairly small number of trials, such as $n=1000$. However, in our computer simulation, described in the following section, we used $10^{5}$ trials in each experiment.

\section{Computer Simulations}

We tested the theoretical results obtained in the previous section by performing computer experiments on randomly throwing balls into two bins. These experiments were carried out using simple Monte Carlo simulations [4], [5].

Table 1. Monte Carlo Simulation Results of Throwing $N$ Balls into Two Bins with Probabilities $p$ for Bin Number 1 and 1- $p$ for Bin Number 2. $N^{*}$ and $p^{*}$ Are the Estimated Values Obtained from the Simulation Data

\begin{tabular}{|c|c|c|c|c|c|}
\hline$N$ & $p$ & $\left\langle N_{1}-N_{2}\right\rangle$ & $\left\langle\left(N_{1}-N_{2}\right)^{2}\right\rangle$ & $N^{*}$ & $p^{*}$ \\
\hline 10 & .2 & -5.9939 & 42.3308 & 10.0 & 200 \\
\hline 2 & .2 & -11.9916 & 156.5482 & 20.0 & .200 \\
\hline 3 & .2 & -17.9799 & 342.3673 & 29.9 & .199 \\
\hline 4 & .2 & -23.9858 & 600.8974 & 40.0 & .200 \\
\hline 5 & .2 & -29.9970 & 931.8307 & 50.0 & .200 \\
\hline 6 & .2 & -35.9977 & 1334.2660 & 60.0 & .200 \\
\hline 7 & .2 & -42.0009 & 1808.8239 & 70.0 & .200 \\
\hline 8 & .2 & -48.0071 & 2355.7242 & 79.9 & .200 \\
\hline 9 & .2 & -54.0017 & 2973.7854 & 90.0 & .200 \\
\hline 1 & $0 \quad .2$ & -59.9856 & 3662.3679 & 100.1 & .200 \\
\hline 10 & .5 & .0025 & 10.0772 & 10.1 & .500 \\
\hline 20 & .5 & -.0020 & 20.2251 & 20.2 & .500 \\
\hline 30 & .5 & -.0006 & 30.3178 & 30.3 & .500 \\
\hline 40 & .5 & .0018 & 40.2820 & 40.3 & .500 \\
\hline 50 & .5 & -.0090 & 50.3777 & 50.4 & .500 \\
\hline 60 & .5 & -.0141 & 60.2753 & 60.3 & .500 \\
\hline 70 & .5 & -.0292 & 70.2711 & 70.3 & .500 \\
\hline 80 & .5 & -.0416 & 80.1803 & 80.2 & .500 \\
\hline 90 & .5 & -.0477 & 90.0617 & 90.1 & .500 \\
\hline 100 & .5 & -.0265 & 100.0306 & 100.0 & .500 \\
\hline
\end{tabular}
of Columns 3 and 4 
The details of our Monte Carlo simulations are as follows: At the beginning of the experiment, the number of balls in each bin is set equal to zero, i.e., $N_{1}=N_{2}=0$. An arbitrary probability $p$ is chosen for bin number 1 (such as $p=0.2$ ). We now want to throw a ball into the bins with probability $p$ of going into bin number 1 and probability 1- $p$ of going into bin number 2 . To do so, we generate a random number $r$ such that $0 \leq \mathrm{r}<1$ and compare it to $p$. If $r \leq p$, the number of balls in bin 1 is increased by 1 , i.e., we set $N_{1}=N_{1}+1$, otherwise we set $N_{2}=N_{2}+1$. We repeat this process $N$ times, so that a total of $N$ balls are thrown into the bins, each with probability $p$ of going into bin number 1 and probability 1- $p$ of going into bin number 2 . At the end, the number of balls in each bin is counted and the quantities $N_{1}-N_{2}$ and $\left(N_{1}-N_{2}\right)^{2}$ are calculated and saved. This constitutes one trial. The trial is repeated $10^{5}$ times and average quantities $\left\langle N_{1}-N_{2}\right\rangle$ and $\left\langle\left(N_{1}-N_{2}\right)^{2}\right\rangle$ are calculated.

We repeated the above experiment with different number of balls $\mathrm{N}$. We also repeated the experiment with $p=0.5$ and different number of balls. The results of these experiments are compiled in Table 1 . The first and the second columns of the table show the values of $N$ and $p$ used in our Monte Carlo simulations. The fifth and the sixth columns show the vales of $N$ and $p$ that are calculated using the simulation data $<N_{1}-N_{2}>$ and $<\left(N_{1}-N_{2}\right)^{2}>$ and equations (8) and (9). We have denoted these by $N^{*}$ and $p^{*}$.

Table 2. Monte Carlo Simulation Results of Throwing $N$ Balls into Two Bins with Variable Probabilities. $N^{*}$ and $p^{*}$ Are the Estimated Values Obtained from the Simulation Data of Columns 2 and 3, Respectively

\begin{tabular}{rrrrl}
\hline \hline$N$ & $\left\langle N_{1}-N_{2}\right\rangle$ & $\left\langle\left(N_{1}-N_{2}\right)^{2}\right\rangle$ & \multicolumn{1}{l}{$N^{*}$} & \multicolumn{1}{l}{$p^{*}$} \\
\hline \hline 1 & .0007 & 1.0000 & 1.0 & .500 \\
\hline 2 & .0010 & 2.6605 & 2.0 & .500 \\
\hline 3 & .0008 & 4.9860 & 3.0 & .500 \\
\hline 4 & -.0039 & 7.9724 & 4.0 & .500 \\
\hline 5 & -.0067 & 11.6229 & 5.0 & .500 \\
\hline 6 & -.0064 & 15.9376 & 6.0 & .500 \\
\hline 7 & -.0041 & 20.9157 & 7.0 & .500 \\
\hline 8 & .0035 & 26.5491 & 8.0 & .500 \\
\hline 9 & .0003 & 32.8286 & 9.0 & .500 \\
\hline 10 & -.0039 & 39.7886 & 10.0 & .500 \\
\hline 11 & -.0035 & 47.3748 & 11.0 & .500 \\
\hline 12 & -.0041 & 55.7011 & 12.0 & .500 \\
\hline 13 & .0004 & 64.7038 & 13.0 & .500 \\
\hline 14 & .0005 & 74.3227 & 14.0 & .500 \\
\hline 15 & .0035 & 84.5828 & 15.0 & .500 \\
\hline 16 & .0008 & 95.6012 & 16.0 & .500 \\
\hline 17 & .0017 & 107.2562 & 17.0 & .500 \\
\hline 18 & .0013 & 119.4703 & 18.0 & .500 \\
\hline 19 & -.0011 & 132.4702 & 19.0 &
\end{tabular}

Finally, we performed Monte Carlo simulations in which we changed the probability $p$ randomly from trial to trial. The probability $p$, however, was uniformly distributed between 0 and 1 . In this case, therefore, the mean probability is 0.5 and the value of $\left\langle N_{1}-N_{2}\right\rangle$ according to equation (4) becomes zero. However, the value of $\left\langle\left(N_{1}-N_{2}\right)^{2}>\right.$ from equation (7) does not reduce to $N$ because in general $<f(p)>\neq f(<p>)$. Instead, the average value of the function $(2 p-1)^{2}$ in equation (7) should be calculated according to the correct method of finding the average of a function over an interval [6],

$$
\langle f(x)\rangle=\frac{1}{b-a} \int_{a}^{b} f(x) d x
$$

Therefore,

$$
<(2 p-1)^{2}>=\int_{0}^{1}(2 p-1)^{2} d p=\frac{1}{3}
$$


Substituting this in equation (7) gives

$$
\left\langle\left(N_{1}-N_{2}\right)^{2}\right\rangle=\frac{N(N+2)}{3}
$$

which is a quadratic equation and can be solved for $N$,

$$
N=-1+\sqrt{1+3<\left(N_{1}-N_{2}\right)^{2}>}
$$

Therefore, again $N$ can be calculated from $\left\langle\left(N_{1}-N_{2}\right)^{2}>\right.$. Table 2 shows the results of the simulations and the values of $N$ that are estimated from this equation. Again we reiterate that in this case $\langle p>=0.5$ and $<N_{1}-N_{2}>=0$.

\section{Conclusion}

Despite the fact that the sum and the difference of two numbers are independent from each other and one cannot be obtained from the other, when a number is randomly divided into two parts, such as randomly throwing balls into two bins, their difference $N_{1}-N_{2}$ provides a very small amount of latent information about their sum $N_{1}+N_{2}$. The information here is that for non-negative numbers $N_{1}$ and $N_{2}$ we have $\left|N_{1}-N_{2}\right| \leq$ $N_{1}+N_{2}$, in which the equality sign holds only if all the balls go into one bin. Therefore, for a sufficiently large number of trials, $\max \left(\left|N_{1}-N_{2}\right|\right)$ provides an estimate of $N_{1}+N_{2}$, which statistically becomes more and more accurate as the number of trials increases.

In principle the above algorithm produces an estimate of the sum of two numbers from their difference. However, unless the numbers are small, the process is very inefficient as it requires an extremely large number of trials. On the other hand the method suggested in this article, which utilizes the mean value of $N_{1}-N_{2}$ and its variance, provides a very efficient algorithm which yields a highly accurate estimate of the sum of two numbers from their difference as well as the probabilities of the process even with a fairly small number of trials.

In summary, using a statistical analysis and Monte Carlo simulations, we have shown how a very small amount of information imbedded in a single random or stochastic event can accumulated over a large number of trials to produce a significant amount of information on some of the parameters of the system. The information thus accumulated becomes more and more accurate as the number of trials increases. Although in this work we have limited our attention to finding the sum of two numbers from their difference, the analysis presented here provides motivation for further investigation and accumulating and extracting information in other random processes.

\section{References}

[1] Spitzer, F. (1964). Principles of random walk. Graduate Texts in Mathematics. New York: Springer-Verlag.

[2] Malkiel, B. G. (2016). A Random Walk Down Wall Street. New York: Norton \& Company, Inc.

[3] Gould, H., \& Tobochnik, J. (1996). An Introduction to Computer Simulation Methods. Applications to Physical Systems (2nd ed.). Reading, Massachusetts: Addison-Wesley. p. 194.

[4] Landau, D. P., \& Binder, K. (2015). A Guide to Monte Carlo Simulations in Statistical Physics (4th ed.). Cambridge, UK: Cambridge University Press.

[5] Binder, K., \& Heermann, D. W. (1992). Monte Carlo Simulation in Statistical Physics. An Introduction (2nd corrected ed.). New York: Springer-Verlag. p. 68

[6] Larson, R., \& Edwards, B. (2015). Calculus of a Single Variable: Early Transcendental Functions (6th ed.). Boston, Massachusetts: Cengage Learning. p. 317. 


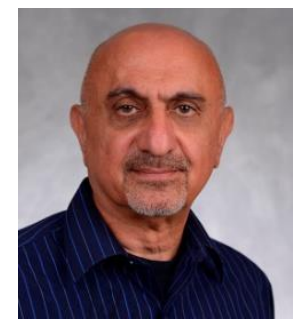

Carlo simulations.

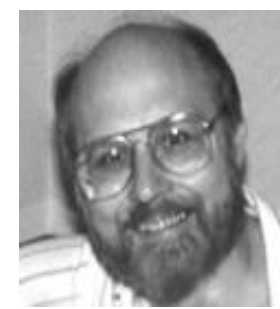

Pirooz Mohazzabi received his $\mathrm{PhD}$ in materials science and engineering from the University of California, Berkeley, in 1975. He joined the University of Wisconsin, Parkside in 1986, where he is currently a professor of physics. He has published extensively in a wide variety of areas, ranging from bicycle stability to cancer theory. His current research focuses on thermodynamics and statistical mechanics of small systems, using theoretical and computational methods, including molecular dynamics and Monte

Thomas A. Fournelle was a professor of mathematics at the University of Wisconsin-Parkside. He was an excellent mathematician and educator with a deep understanding of the subject. His areas of interest included infinite group theory, mathematical modeling, and numerical methods. Tom received several teaching awards and was widely acknowledged by his students as the best teacher they ever had. He has been deeply missed by the department since his untimely passing in 2010 . 